\title{
Quantitative Nonlinear Analysis of Autocatalytic Pathways with Applications to Glycolysis
}

\author{
Gentian Buzi, Ufuk Topcu, and John C. Doyle
}

\begin{abstract}
Autocatalytic pathways are frequently encountered in biological networks. One such pathway, the glycolytic pathway, is of special importance and has been studied extensively. Using tools from linear systems theory, our previous work on a simple two dimensional model of glycolysis demonstrated that autocatalysis can aggravate control performance and contribute to instability. Here, we expand this work and study properties of nonlinear autocatalytic pathway models (of which glycolysis is an example). Changes in the concentration of metabolites and catalyzing enzymes during the lifetime of the cell can perturb the system from the nominal operating point of the pathway. We investigate effects of such perturbations through the estimation of invariant subsets of the region of attraction around nominal operating conditions (i.e., a measure of the set of perturbations from which the cell recovers). Numerical experiments demonstrate that systems that are robust with respect to perturbations in parameter space have easily "verifiable" region of attraction properties in terms of proof complexity.
\end{abstract}

\section{INTRODUCTION}

In cells, core metabolism is comprised of metabolic reaction networks (pathways), which are sequences of chemical reactions usually connected in series. These chemical reactions are catalyzed by specialized proteins called enzymes, which speed up (catalyze) the reactions [1]. The cell uses these enzymes to regulate (control) the metabolic pathways via two distinct mechanisms, transcriptional regulation and allosteric regulation. Transcriptional regulation changes the concentration levels of the enzymes by controlling gene expression, thus speeding up or slowing down the corresponding reactions. Allosteric regulation is the process by which a molecule binds to an enzyme causing the enzyme to change shape and become more effective (activation) or less effective (inhibition). It happens at a much faster timescale than transcriptional regulation because the effects of binding are immediate.

Some of the metabolic pathways contain reactions that require the consumption of one of their own products, thus creating a positive feedback loop. Such pathways are called autocatalytic pathways. They are very common in biology; indeed at a certain level all biological networks are massively autocatalytic, since in every cell, food and resources are broken down to create energy and components via processes that also require the use of those same components and energy.

The simplest and most widely studied autocatalytic pathway is glycolysis. The cell relies on this metabolic pathway

The authors are with the Control and Dynamical Systems, California Institute of Technology, Pasadena, CA, 91125, USA genti, utopcu, doyleecds.caltech.edu to produce energy anaerobically (without oxygen) by breaking down glucose. In glycolysis, the autocatalysis happens when two ATP molecules (energy carriers) are consumed early in the pathway (in this process Hexokinase (HK) and Phosphofructokinase (PFK) act as the catalyzing enzymes). Four ATP molecules are generated later in the pathway, for a net return of two ATP molecules (Fig. 1). One of the catalyzing enzymes, PFK, plays a crucial role since it is regulated by ATP (the output of the pathway). PFK is inactive when ATP concentration is high [2], and this form of inhibition is the mechanism used to stabilize the pathway on the fast time-scale.

Using tools from linear systems theory, our previous work on a simple two dimensional model of glycolysis demonstrated that autocatalysis can aggravate control performance and contribute to instability [3]. However, the behavior of the system away from the equilibrium points may not be well characterized by such tools. Therefore, in order to reason about behavior due to large perturbation, it is of interest to develop an understanding of the system behavior away from equilibrium points as well as to obtain a more global picture of the system. To this end, in the present work, we study the nonlinear properties of the autocatalytic metabolic networks, of which the inner autocatalytic loop of glycolysis is a subset (highlighted part in Fig. 1, bottom right). These networks are composed of a chains of reactions that convert one metabolite to another, ultimately producing copies of the final product of the pathways (ATP in the case of glycolysis). These pathways also require the consumption of copies of their final product to convert the pathway input into the first metabolite (see (1)).

Linear analysis shows that negative feedback via product inhibition can stabilize these pathways. However changes in the concentration of catalyzing enzymes during the lifetime of the cell lead to changes in the reaction rates and these changes will perturb the system from the equilibrium point. With these changes in the cell's operating conditions two questions naturally arise; Can the controller restore the system to normal operating conditions from these perturbations? How big can these perturbations from equilibrium be before the system crashes? In essence we are asking questions regarding the size of the region of attraction (RoA) of the equilibrium point. In this paper, we study such questions using recently developed nonlinear system analysis tools based on Lyapunov type characterizations of invariant subsets of the RoA [4] and numerical optimization (especially Sumsof-squares programming) [5]. 


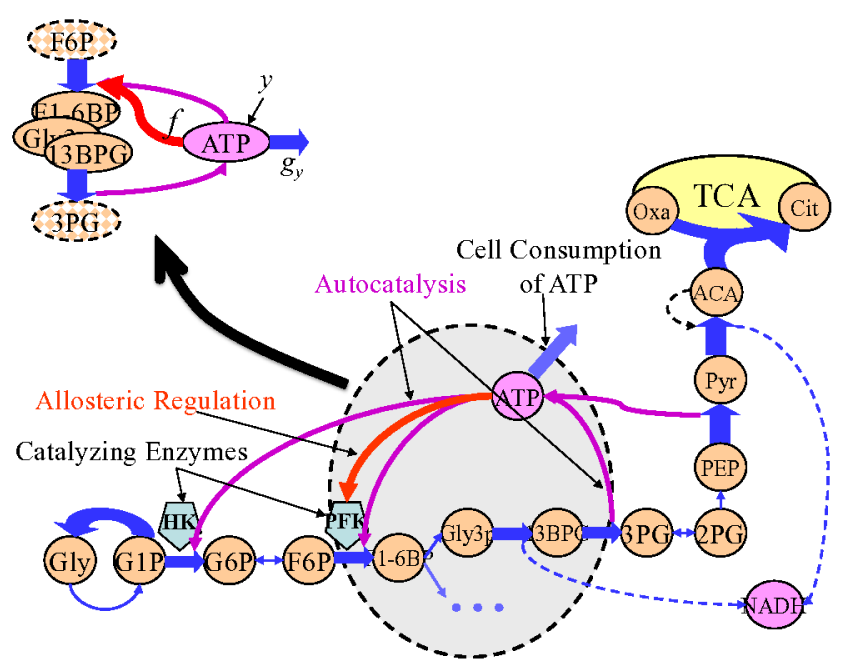

Fig. 1. Glycolysis pathway (bottom right) and reduction of the inner autocatalytic loop of the pathway to a lower dimensional model (top left).

\section{AN AUTOCATALYTIC PATHWAY MODEL}

Consider the autocatalytic metabolic pathway with multiple intermediate metabolite reactions

$$
\begin{aligned}
& u+a y \rightarrow f x_{1} \\
& x_{1} \rightarrow g_{1} x_{2} \cdots \rightarrow^{g_{n-1}} x_{n} \rightarrow^{g_{n}}(a+b) y+x_{n+1} \\
& y \rightarrow g_{y} \phi \text {. }
\end{aligned}
$$

Here, $u$ is some precursor and source of energy (e.g. F6P in Fig. 1) with no dynamics associated, $y$ denotes the product of the pathway (e.g. ATP), $x_{i}$ are intermediate metabolites (e.g. F1-6BP, Gly3p, and 3BPG), $\phi$ is a null state, $a$ is the number of ATP molecules invested in the pathway, and $a+b$ is the number of ATP molecules produced. $A \rightarrow B(A \rightarrow f$ $B$ ) denotes a chemical reaction that converts the chemical species $A$ to the chemical species $B$ (at rate $f$ ). As mentioned earlier, the above reactions are catalyzed by enzymes. We do not show these enzymes explicitly in the above reactions, but note that the rates of the chemical reactions depend on their concentrations.

The inner autocatalytic loop of glycolysis (Fig. 1), has the same topology as system 1 . In this case, the first reaction in the pathway is catalyzed by PFK and the rate of this reaction is modeled by $f(y)$, which is directly related to PFK's activity. A reasonable form for $f(y)$ that also agrees with experimental data is $f(y)=\frac{V y^{q}}{1+\gamma y^{h}}$ [2], [6], where $q$ is positive and captures the strength of autocatalysis and $\gamma$ are $h$ are determined by the enzyme and regulate the strength of feedback inhibition. $V$ is related to the availability of precursors such as F6P.

The corresponding ordinary differential equations in terms of the stoichiometry matrix $S$ and reaction fluxes is

$$
\left[\begin{array}{c}
\dot{x}_{1} \\
\dot{x}_{2} \\
\vdots \\
\dot{x}_{n} \\
\dot{y}
\end{array}\right]=S\left[\begin{array}{c}
f(y) \\
g_{1}\left(x_{1}\right) \\
\vdots \\
g_{n-1}\left(x_{n-1}\right) \\
g_{n}\left(x_{n}\right) \\
g_{y}(y)
\end{array}\right]
$$
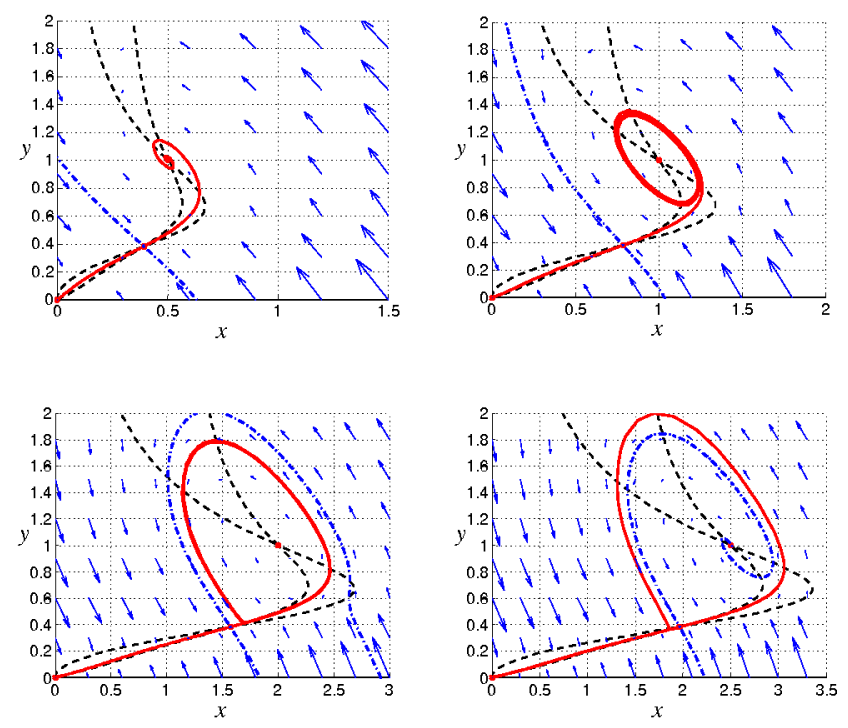

Fig. 2. Changes in the dynamic behavior due to changes in the reaction rate $k$.

for $x_{i} \geq 0, y \geq 0$. Here,

$$
S:=\left[\begin{array}{cccccc}
1 & -1 & & 0 & 0 & 0 \\
0 & 1 & \ddots & 0 & 0 & 0 \\
\vdots & & \ddots & & \ddots & \vdots \\
0 & 0 & & 1 & -1 & 0 \\
-a & 0 & \cdots & 0 & b+a & -1
\end{array}\right]
$$

$g_{1}, \ldots, g_{n}$, and $g_{y}$ are continuous, monotone, increasing functions that vanish at 0 .

\section{A. Properties of the model}

Throughout the lifetime of the cell the concentrations of the catalyzing enzymes fluctuate. These fluctuations result in changes in the reaction rates in the pathway. Under these perturbations the system can exhibit a very rich dynamic behavior. Fig. 2 illustrates the changes in the behavior of a realization of the system (with $n=1, h=4, \gamma=5, q=2$, $V=6, g_{y}(y)=\frac{1.2 y}{0.2+y}$ and $\left.g_{x}(x)=k x\right)$ due to changes in $k$. For $k=2$, the system is stable around its normal operating point $\left(\frac{1}{k}, 1\right)$ (top left). However, if the concentration of the intermediate catalyzing enzyme decreases, the reaction rate $k$ decreases possibly leading to oscillations or even the system to crash. For example, a reaction rate $k=1$ causes the ATP concentration $(y)$ to oscillate (top right). For a larger decrease, e.g. for $k=0.5$, the oscillations become larger and the system approaches a homoclinic bifurcation, i.e. the limit cycle is close to hitting the saddle point (bottom left). At $k=0.4$ the pathway crashes and the cell dies. The limit cycle opens up as a result of the bifurcation and (almost) all trajectories go to the origin (bottom right).

On the other hand, the system in (2) has some easily analyzable properties. The positive orthant is invariant with respect to the flow of (2). It can be shown that for $h>q$ and under some mild assumptions on $g_{y}$, the trajectories of the system are bounded (see [7]). In addition, the number of 
equilibrium points of (2) in the positive orthant is determined by the number of the solutions of the equation $b f(y)=$ $\left.g_{y}(y)\right)$. Consequently, concentration of ATP at the operating point is determined by the ATP production and consumption rates, and not the intermediate reactions. As a result, there are some simple conditions that characterize crashes in the system. For example, if $b f(y)<g_{y}(y), \forall y>0$, the fixed point at the origin is the only fixed point for the system. In this scenario the pathway is consuming ATP $(y)$ faster than it can produce it. It can be shown (for example using $(a+b)\left(x_{1}+\cdots+x_{2}\right)+y$ as a Lyapunov function) that the origin in this case is globally asymptotically stable, i.e. for all initial conditions the ATP concentration goes to zero and consequently the cell dies. Under more common operating conditions, there are additional fixed points in positive orthant, one of which represents the normal operating point of the pathway. Depending on reaction rates, this fixed point can be stable or undergo a Hopf bifurcation and give rise to a limit cycle as shown in the first example.

Another observation from the example above is that the equilibrium concentrations of the intermediate metabolite $(x)$ depend on the concentration level of the catalyzing enzymes. As such, sudden drops in enzyme concentrations will cause a perturbation in the $x$-direction which moves the system from the equilibrium point. How big can this drop be if the system is to continue to converge to equilibrium? To seek for answers for such questions, we study the properties of the nonlinear system around the equilibrium points through estimating regions of attraction.

\section{Estimation of the Region of AtTRACtion}

Consider the system governed by

$$
\dot{\xi}(t)=F(\xi(t))
$$

where $\xi(t) \in \mathcal{R}^{n}$ is the state vector and $F: \mathbb{R}^{n} \rightarrow \mathbb{R}^{n}$ is such that $F(0)=0$, i.e., the origin is an equilibrium point of (3) and $F$ is locally Lipschitz on $\mathbb{R}^{n}$. Let $\varphi(\xi, t)$ denote the solution to (3) with the initial condition $\varphi(\xi, 0)=\xi$. If the origin is asymptotically stable but not globally attractive, one often wants to know which trajectories converge to the origin as time approaches $\infty$. This gives rise to the following definition of the region of attraction:

Definition 1: The region of attraction of the origin for the system (3) is $\left\{\xi \in \mathbb{R}^{n}: \lim _{t \rightarrow \infty} \varphi(\xi, t)=0\right\}$.

For $\eta>0$ and a function $U: \mathbb{R}^{n} \rightarrow \mathbb{R}$, define the $\eta$-sublevel set of $U$ as $\Omega_{U, \eta}:=\left\{\xi \in \mathbb{R}^{n}: U(\xi) \leq \eta\right\}$. We use the following characterization of the invariant subsets of the RoA.

Lemma 1: Let $\alpha \in \mathbb{R}$ be positive. If there exists a continuously differentiable function $U: \mathbb{R}^{n} \rightarrow \mathbb{R}$ such that

$$
\begin{aligned}
& \Omega_{U, \alpha} \text { is bounded, and } \\
& U(0)=0 \text { and } U(\xi)>0 \text { for all } \xi \in \mathbb{R}^{n} \\
& \Omega_{U, \alpha} \backslash\{0\} \subset\left\{\xi \in \mathbb{R}^{n}: \nabla U(\xi) F(\xi)<0\right\}
\end{aligned}
$$

then for all $\xi \in \Omega_{U, \alpha}$, the solution of (3) exists, satisfies $\varphi(t ; \xi) \in \Omega_{U, \alpha}$ for all $t \geq 0$, and $\lim _{t \rightarrow \infty} \varphi(t ; \xi)=0$, i.e., $\Omega_{U, \alpha}$ is an invariant subset of the RoA.

The estimate (i.e., invariant subset characterized by the conditions in Lemma 1) of the RoA can be enlarged through the optimization problem

$$
\max _{\beta>0, U \in \mathcal{U}} \beta \text { subject to }(4)-(6), \Omega_{\xi^{T} \xi, \beta} \subseteq \Omega_{U, \alpha} \text {. }
$$

Here $\mathcal{U}$ denotes the set of candidate Lyapunov functions over which the maximum is defined, for example all continuously differentiable functions. Following [8], in order to make (11) amenable to SOS programming, we restrict $\mathcal{U}$ to be all polynomials of some fixed degree and use the wellknown SOS sufficient condition for polynomial nonnegativity [5] and simple generalizations of the $S$-procedure [8] to obtain (numerically verifiable) sufficient conditions for the set containment constraints in the optimization problem (7). Let $\mathbb{R}[\xi]$ and $\Sigma[\xi]$ denote the set of polynomials (in $\xi$ ) with real coefficients and the subset of SOS polynomials. Let $\epsilon>0$. Then, the constraint

$$
U-\epsilon \xi^{T} \xi \in \Sigma[\xi]
$$

and $U(0)=0$ are sufficient conditions for (4) and (5). If $s_{1} \in \Sigma[\xi]$, then

$$
-\left[\left(\beta-\xi^{T} \xi\right) s_{1}+(U-\alpha)\right] \in \Sigma[\xi]
$$

implies the set containment $\Omega_{\xi^{T} \xi, \beta} \subseteq \Omega_{U, \alpha}$. Moreover, if $s_{2}, s_{3} \in \Sigma[\xi]$, then

$$
-\left[(\alpha-U) s_{2}+\nabla U F s_{3}+\epsilon \xi^{T} \xi\right] \in \Sigma[\xi]
$$

is a sufficient condition for (6). Using these sufficient conditions, a lower bound on the optimal value of $\beta$ in (7) can be computed through the optimization problem

$$
\begin{gathered}
\max _{U \in \mathcal{U}, \beta, s_{i} \in \mathcal{S}_{i}} \beta \text { subject to }(8)-(10), \\
U(0)=0, s_{i} \in \Sigma[\xi], \beta>0 .
\end{gathered}
$$

Here, the sets $\mathcal{U}$ and $\mathcal{S}_{i}$ are prescribed finite-dimensional subspaces of polynomials. The optimization problem in (11) is bilinear (i.e., includes bilinear matrix inequality constraints) because of the product terms $\beta s_{1}$ in (9) and $U s_{2}$ and $\nabla U F s_{3}$ in (10). However, the problem has more structure than a general bilinear optimization problem: if $U$ is fixed, the problem becomes affine in $s_{1}, s_{2}$, and $s_{3}$ and vice versa. Based on this observation "suboptimal" solutions for (11) can be obtained using a coordinate-wise affine search procedure: (i) for given (fixed) $U$ feasible for (11), maximize $\beta$ over the choice of $s_{1}, s_{2}$, and $s_{3}$ subject to the constraints in (11); (ii) fix $s_{1}, s_{2}$, and $s_{3}$ from the previous step and maximize $\beta$ over the choice of $U$ subject to the constraints in (11); (iii) repeat the previous two steps. Note that the optimization problems in steps (i) and (ii) can be solved as affine semidefinite programs via a line search on scalar decision variables $\alpha$ and $\beta$ [8] using a semidefinite programming solver, e.g. 
SeDuMi [9]. A "feasible" $U$ to initialize this procedure can be obtained through

$$
\begin{gathered}
\max _{U \in \mathcal{U}, \gamma, s_{0} \in \mathcal{S}_{0}} \beta \text { subject to }(8), U(0)=0, s_{0} \in \Sigma[\xi], \beta>0, \\
\nabla U F(\xi)-\epsilon \xi^{T} \xi+s_{0}\left(\xi^{T} \xi-\eta\right) \in \Sigma[\xi] .
\end{gathered}
$$

Let $U_{0}$ and $\eta_{0}$ denote the optimal values of $\eta$ and $U$ in (12) and $\alpha_{0}$ be the largest of $\alpha$ such that $\Omega_{U_{0}, \alpha_{0}} \subseteq \Omega_{\xi^{T} \xi, \eta_{0}}$. Then, $U_{0}$ and $\alpha$ are feasible for (11) ([10]).

The optimization problem in (11) provides a means for computing invariant subsets of RoA for systems with polynomial vector fields. The extension to systems with rational vector fields is straightforward and discussed next for completeness. To this end, let $\bar{\xi}=\left(\bar{x}_{1}, \ldots, \bar{x}_{n}, \bar{y}\right)$ be the fixed point of interest for the dynamics in (2). If $\bar{\xi} \succ 0$, without loss of generality, let $\bar{y}=1$ and perform a change of coordinates such that the fixed point of interest is at the origin. Consider that the transformed system is of the form (3) for $\xi=\left(x_{1}, \ldots, x_{n}, y\right) \in \mathcal{D}$, where $\mathcal{D}:=$ $\left\{\xi \in \mathbb{R}^{n+1}: \xi \succeq-\bar{\xi}\right\}$. Note that the vector field $F$ can be written as $F(\xi)=\frac{G(\xi)}{H(y)}$, where $H(y):=1+\gamma(y+1)^{h}$ and $G$ is appropriately chosen function of $\xi, H(y)>0$ for all $y \geq-1$, and $G(0)=0$.

Lemma 2: Let $U$ be a continuously differentiable function and $\alpha>0$ that satisfy the conditions in (4)-(5) and $\Omega_{U, \alpha} \backslash\{0\} \subset\left\{\xi \in \mathbb{R}^{n+1}: \nabla U(\xi) G(\xi)<0\right\}$. Then, $\Omega_{U, \alpha} \cap \mathcal{D}$ is an invariant subset of the origin for the dynamics in (3).

Proof: For all $\xi \in \Omega_{U, \alpha} \cap \mathcal{D}, \nabla U(\xi) F(\xi)=$ $\frac{1}{H(y)} \nabla U(\xi) G(\xi)<0$ where the inequality follows from the hypothesis. Since $\mathcal{D}$ is invariant under the flow of (3) and $U$ and $\alpha$ satisfy the conditions in (4)-(6), $\Omega_{U, \alpha} \cap \mathcal{D}$ is an invariant subset of the RoA of the origin for (3).

Example 1: For the system (2), with $n=1$, estimates of the RoA of the fixed point at $y=1$ are shown in Figure 3 for $V=3, h=6, q=1, \gamma=1, g_{1}(x)=1.2 x$ and $g_{y}(y)=y$ (left) and $V=3, h=6, q=2, \gamma=2, g_{1}(x)=1.1 x$ and $g_{y}(y)=y$ (right), using polynomial Lyapunov functions of different degrees. In both cases the closest fixed point (marked by a red dot) limits the maximum size of the RoA estimate.
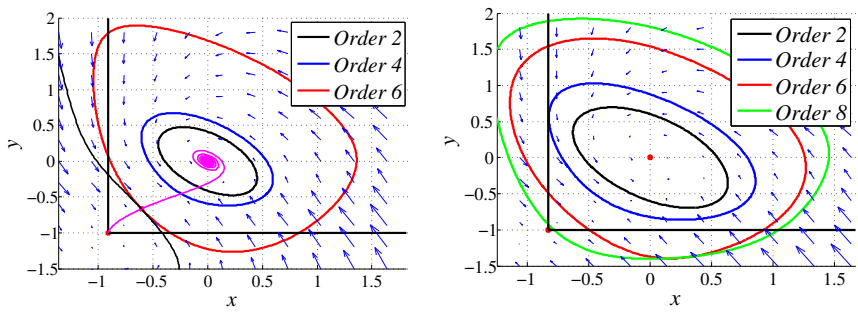

Fig. 3. Higher degree polynomial Lyapunov functions improve the estimated RoA, but this improvement is limited by how close the nearest fixed point is.

\section{Results}

For the rest of this paper we will use special forms of the reaction rates $g_{y}(y)=k_{y} y$ and $g_{i}\left(x_{i}\right)=k_{i} x_{i}$ for $i=$
$1, \ldots, n$. These types of reaction rates appear when massaction kinetics are used to model the reaction rates. We also set the parameters $q=1, a=1$, and $b=1$.

\section{A. Single intermediate reaction}

Consider the dynamics for the case of a single intermediate reaction (i.e., $n=1$ )

$$
\begin{aligned}
& \dot{x}=\frac{(1+\gamma)(y+1)}{1+\gamma(y+1)^{h}}-1-k x \\
& \dot{y}=2 k x-\frac{(1+\gamma)(y+1)}{1+\gamma(y+1)^{h}}+1-y
\end{aligned}
$$

in the domain $\mathcal{D}=\left\{(x, y) \in \mathbb{R}^{2}:(x, y) \succeq\left(-\frac{1}{k},-1\right)\right\}$. This case corresponds to a simple two-state model of glycolysis depicted in Fig. 1 (top left). Here, the operating fixed point has been shifted to the origin and the time is scaled so that $g_{y}(y)=y$. For a fixed $k$, the models generated by the different values of $\gamma$ and $h$ have the same topology in $\mathcal{D}$, i.e., they have fixed points only at $\left(-\frac{1}{k},-1\right)$ and $(0,0)$. For the values of $\gamma$ and $h$ that result in stable linearized dynamics around the origin, we can show that the RoA of the origin is $\mathcal{D} \backslash\left\{\left(-\frac{1}{k},-1\right)\right\}$. Here, we report results for the quantitative local analysis of the glycolysis dynamics for this special case in order to examine the effectiveness of the optimizationbased formulations discussed in the previous section. We investigate the complexity and robustness properties of the systems using two "measures" of complexity as discussed next.

- For each $k$, define the set

$$
\mathcal{B}_{k}:=\left\{(x, y):\left(x^{2}+y^{2}\right)^{1 / 2}<\frac{1}{2} \sqrt{1+\frac{1}{k^{2}}}\right\},
$$

i.e., the ball of radius half the distance to the fixed point at $\left(-\frac{1}{k},-1\right)$. The first measure of complexity is defined as the degree of the smallest degree polynomial Lyapunov function which certifies $\mathcal{B}_{k}$ to be in the RoA. Define the parameter $\hat{h}:=\frac{\gamma}{\gamma+1} h$ that captures the strength of feedback in the linearized dynamics (see [3] for a detailed discussion on $\hat{h}$ ). It can be shown that the linearized dynamics (in the case considered in this subsection) are stable for $0<\hat{h}<k+2$ for all positive values of $k$ [7]. For a given $k$, we set $h$ to the smallest integer greater than or equal to $k+3$ and $\gamma=\hat{h} /(h-\hat{h})$ (to ensure that the degree of the vector field is an integer). For the ranges of parameters $k \in[0.1,5]$ and $\hat{h}$ that lead to asymptotically stable linearized dynamics, we estimate the RoA of the origin using 2nd and 4th degree polynomial Lyapunov functions.

We solve the problem in (11) on a $50 \times 50$ parameter grid over $\{(k, \hat{h}): k \in[0.1,5], 0 \leq \hat{h} \leq k+2\}$ uniform in each of the $k$ and $\hat{h}$ directions. Fig. 4 shows that systems away from the stability boundary $\{(k, \hat{h}): k \in$ $[0.1,5], \hat{h}=k+2\}$ in the parameter space only require a 2nd degree Lyapunov function to verify that $\mathcal{B}_{k}$ is in the RoA, while higher order polynomials are required for many of the systems near the boundary.

- The second measure of complexity is obtained by fixing the degree of the polynomial Lyapunov function candidates 

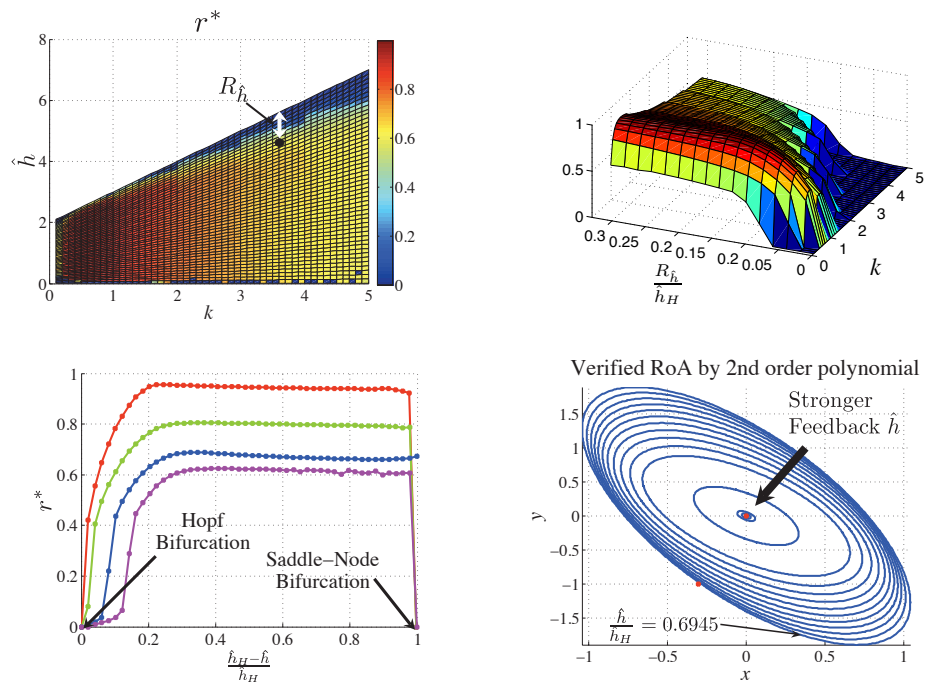

Fig. 5. $\quad \hat{h}_{H}$ is the value of $\hat{h}$ at the Hopf bifurcation. $r^{*}$ is the normalized radius of the RoA estimate, i.e., the maximum value of $r$ such that $\mathcal{B}(r)$ is verified to be in the RoA normalized by the distance $\left(1+1 / k^{2}\right)^{1 / 2}$ to the closest fixed point. The grid on $k-\hat{h}$ parameter region is as indicated above. The curves in the bottom left figure are for $k=1$ (red), 2, 3.3, 4.7 (magenta). Verified invariant subsets of the RoA for $k=3.3$ (bottom right).

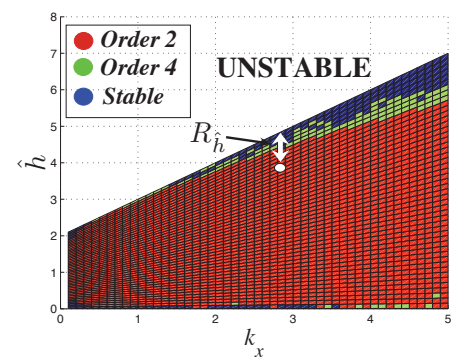

Fig. 4. The set of parameters for which a 2nd degree Lyapunov function verifies that the set $\mathcal{B}_{k}$ is in the RoA (red), the set that needed a 4th degree Lyapunov function (green), and the region marked by blue corresponds to linearly asymptotically stable systems that not be verified by a 2 nd or 4 th degree polynomial Lyapunov function (through the procedure outlined in the previous section). For a given value of $k, R_{\hat{h}}$ is the distance to the stability boundary in the parameter space (in the $\hat{h}$ direction).

and comparing the size of the RoA verified by that polynomial. As a measure of the size of the RoA verified, we will use maximum value of the radius $r$ of the the ball $\mathcal{B}(r):=\left\{(x, y) \mid x^{2}+y^{2}<r^{2}\right\}$ such that $\mathcal{B}(r) \subset \Omega_{U^{*}, \alpha^{*}}$, where $U^{*}$ and $\alpha^{*}$ are the optimal values from (11). Fig. 5 summarizes the results obtained using quadratic Lyapunov functions for the values of $k$ and $\hat{h}$ in the ranges indicated above. The main observation is that as $\hat{h}$ gets large and close to the Hopf bifurcation value (i.e., the feedback strength increases), the radius of the verified RoA gets smaller.

\section{B. Multiple intermediate reactions}

We now apply the RoA analysis to autocatalytic pathways with multiple intermediate reactions (specifically with 2 and 3 intermediate reactions). In the model of glycolysis with a single intermediate reaction, the state $y$ corresponds to the level of ATP and the other state $x_{1}$ can be considered as a summary of the reactions in the dotted circle in the bottom right corner of Fig. 1 (obtained through model reduction procedures [6]). In the models with multiple intermediate reactions, the new (intermediate) states can be considered to capture the intermediate reactions in a detailed manner.
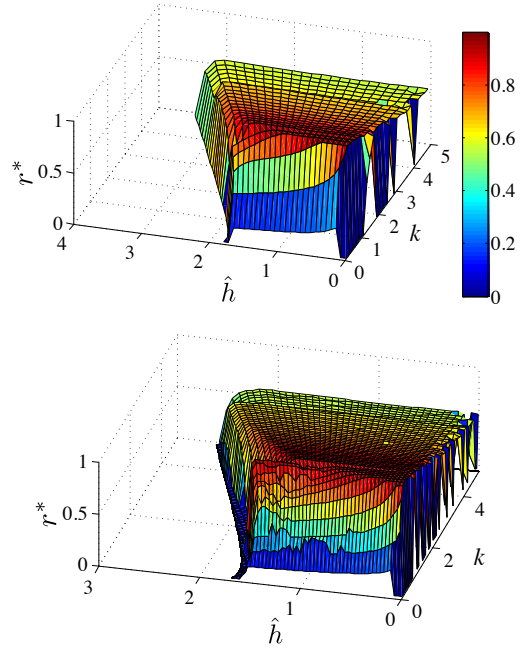

Fig. 6. $r^{*}$ is the normalized radius of the RoA estimate, i.e., the maximum value of $r$ such that $\mathcal{B}(r)$ is verified to be in the RoA normalized by the distance $\sqrt{1+n / k^{2}}, n=2,3$, to the nearest fixed point (top for $n=2$ and bottom for $n=3$ ).

As in the case $n=1$ (i.e., two states), 2nd degree polynomial Lyapunov functions verify large subsets of the RoA of the origin for a wide range of parameter values. For three-state and four-state systems with $g_{i}\left(x_{i}\right)=k x_{i}, q=1$, $g_{y}(y)=y$, Fig. 6 shows the radius of RoA estimate normalized by the distance $\sqrt{1+n / k^{2}}$ to the closest fixed point. It shows that for the majority of the parameter values, the radius of the RoA estimate is at least half the actual RoA radius (measured as the distance from the origin to the equilibrium points at $(-1 / k,-1 / k,-1)$ and $(-1 / k,-1 / k,-1 / k,-1)$ for $n=2$ and 3 respectively). Similar to the two-state case the parameter regions where the estimate is not as good is close to the stability boundary. 
The "size" of the optimization problem in (11) grows with the length of the pathway (number of states in (3)), the degree of the numerator and denominator of $F$ (i.e., $q$ and $h$ ), and the degree of the Lyapunov function candidates. This growth may render the SOS programming based analysis impractical for even modest length pathways (see [11] for a more detailed discussion). We therefore limit our analysis to small dimensional state space models. For larger dimensional systems we emphasize that exploiting the underlying structure of the pathway (i.e., a chain of intermediate reaction coupled with a autocatalytic component) leads to more efficient compositional analysis tools (e.g., see [12]).

\section{Discussion AND CONCLUSIONS}

The optimization-based RoA analysis of the models of autocatalytic pathways for glycolysis show that the RoA of the fixed point where the cell operates is "large," which means that glycolysis is not sensitive to perturbation in the concentrations of the products and reactants (i.e., perturbation in the state space). It also reveals that low degree polynomial Lyapunov functions can verify large sets in the state space to be invariant subsets of the RoA for a wide range of parameters. Moreover, as seen in Figures 5 (top right) and 6 it is easier to verify large invariant subsets of the RoA for systems that are robust to parameter variations (i.e., those in the "middle" of the stability region in the parameter space). On the other hand, comparable size invariant subsets of the RoA for systems that fall closer to the stability boundary can be verified by polynomial Lyapunov functions of degree larger than 2 (see Fig. 4). These systems are clearly not robust to parameter variations as small changes in parameters may result in instability.

We define the complexity of a realization of model (13) by the complexity of the vector field in the neighborhood of the operating equilibrium point. We then develop two related approaches of quantifying this view of complexity by using either the smallest order polynomial Lyapunov function needed to verify a specific set as an invariant subset of the RoA or the size of the RoA verified by a specific order polynomial Lyapunov function. We compare realizations that are "topologically equivalent," i.e., realizations that have the same number and location of fixed points. Based on these measures of complexity, it can be concluded that "complex systems" are those closer to the stability boundary in the parameter space. This observation also suggests that the socalled "complexity implies fragility" notion exists for the autocatalytic pathways models studied here [13]. Namely, systems characterized as complex by the measures utilized here are also fragile, i.e., small perturbations in $\hat{h}$ causes significant changes in the system behavior. For example, in the results reported in the section IV a normalization is used so that the extent of the actual RoA is the same for all (stabilizing) values of $\hat{h}$ for a fixed $k$. However, small changes in $\hat{h}$ (for fixed $k$ ) close to the stability boundary lead to several different regimes: (i) simple polynomial Lyapunov functions certify large invariant subsets of the RoA; (ii) simple polynomial Lyapunov functions can only certify (relatively) smaller sets to be in the RoA; (iii) simple polynomial Lyapunov functions cannot certify (to the numerical tolerances used in the numerical computations) any invariant subset of the RoA.

Overall, the global analysis of the model in (13) suggests that oscillations and crashes in glycolysis are not caused by perturbations in state space (i.e., perturbations in the concentrations of the products and reactants), but rather perturbations in the parameter space (i.e., catalytic enzyme concentrations, precursors to the pathway, ATP demand, or temperature) as observed in the literature [14], [15], [16], [17], [18], [19].

\section{REFERENCES}

[1] B. Alberts, A. Johnson, J. Lewis, M. Raff, K. Roberts, and P. Walter, Molecular Biology of the Cell, Fourth Edition. Garland, 2002.

[2] M. Banuelos, C. Gancedo, and J. M. Gancedo, "Activation by phosphate of yeast phosphofructokinase." J Biol Chem, vol. 252, no. 18, pp. 6394-6398, 1977.

[3] F. A. Chandra, G. Buzi, and J. C. Doyle, "Linear control analysis of the autocatalytic glycolysis system," in Proc of the American Control Conference, 2009, pp. 319-324.

[4] M. Vidyasagar, Nonlinear Systems Analysis, $2^{\text {nd }}$ ed. Prentice Hall, 1993.

[5] P. Parrilo, "Semidefinite programming relaxations for semialgebraic problems," Mathematical Programming Series B, vol. 96, no. 2, pp. 293-320, 2003.

[6] S. Dano, M. F. Madsen, H. Schmidt, and G. Cedersund, "Reduction of a biochemical model with preservation of its basic dynamic properties," FEBS Journal, vol. 273, no. 21, 2006.

[7] G. Buzi, "Control theoretic analysis of autocatalytic networks in biology with applications in glycolysis," Ph.D. Dissertation, California Institute of Technology, 2010.

[8] U. Topcu, A. Packard, and P. Seiler, "Local stability analysis using simulations and sum-of-squares programming," Automatica, vol. 44, pp. $2669-2675,2008$.

[9] J. F. Sturm, "Using SeDuMi 1.02, a MATLAB toolbox for optimization over symmetric cones," Optimization Methods and Software, vol. 1112, pp. 625-653, 1999.

[10] U. Topcu and A. Packard, "Linearized analysis versus optimizationbased nonlinear analysis for nonlinear systems," in Proc. of the American Control Conference, 2009, pp. 790-795.

[11] — , "Local stability analysis for uncertain nonlinear systems," IEEE Transactions on Automatic Control, vol. 54, pp. 1042-1047, 2009.

[12] G. Buzi, U. Topcu, and J. C. Doyle, "Compositional analysis of autocatalytic networks in biology," in Proc. of the American Control Conference, 2010.

[13] D. Gayme, M. Fazel, and J. C. Doyle, "Complexity in automation of SOS proofs: an illustrative example," in Proc. of the Conference on Decision and Control, 2006, pp. 5851-5856.

[14] B. Hess and A. Boiteux, "Substrate control of glycolytic oscillations," in Biological and Biochemical Oscillators, B. Chance, E. K. Pye, A. A.K. Ghosh, and B. Hess, Eds. Academic Press, 1973, pp. 229241.

[15] M. Bier, B. Teusink, B. N. Kholodenko, and H. V. Westerhoff, "Control analysis of glycolytic oscillations." Biophys Chem, vol. 62, pp. 15-24, 1996.

[16] K. Nielsen and P. G. Sorensen, "Sustained oscillations in glycolysis: an experimental and theoretical study of chaotic and complex periodic behavior and quenching of simple oscillations." Biophysical Chemistry, vol. 72, pp. 49-62, 1998.

[17] P. Richard, "The rhythm of yeast," FEMS Microb Rev, vol. 27, pp. 547-557, 2003.

[18] P. Ruoff, M. Christensen, J. Wolf, and R. Heinrich, "Temperature dependency and temperature compensation in a model of yeast glycolytic oscillations." Biophysical Chemistry, vol. 106, pp. 179-192, 2003.

[19] B. Teusink, B. M. Bakker, and H. V. Westerhoff, "Control of frequency and amplitudes is shared by all enzymes in three models for yeast glycolytic oscillations." Biochem Biophys Acta, vol. 1275, pp. 204212, 1996. 